\title{
Preparation of Graphene-Modified Anticorrosion Coating and Study on Its Corrosion Resistance Mechanism
}

\author{
Peng Wang $(\mathbb{D}$ and Dayong Cai \\ College of Materials Science and Engineering, Yanshan University, Qinhuangdao 066004, China \\ Correspondence should be addressed to Peng Wang; lrwp@ysu.edu.cn
}

Received 13 August 2020; Revised 15 October 2020; Accepted 16 October 2020; Published 2 November 2020

Academic Editor: Jinlong Liu

Copyright ( 2020 Peng Wang and Dayong Cai. This is an open access article distributed under the Creative Commons Attribution License, which permits unrestricted use, distribution, and reproduction in any medium, provided the original work is properly cited.

\begin{abstract}
When aluminum alloy is present in a $\mathrm{Cl}^{-}$-rich environment, the surface oxide film is easily damaged, resulting in faster dissolution of the substrate. The application of graphene-modified anticorrosion coating can effectively prevent the occurrence of corrosion. In this study, to explore the corrosion resistance of graphene-modified anticorrosion coating on the surface of aluminum alloy, we prepared graphene-modified anticorrosion coating on the surface of aluminum alloy and investigated the corrosion resistance mechanism. Epoxy resin primer and polyurethane top coat were modified by predispersed reduced graphene oxide (rGO). Scanning electron microscope (SEM) and Raman spectrum were used to investigate the microstructure of graphene-modified anticorrosion coating, and it was found that the addition of rGO could effectively improve the porosity defect of epoxy resin primer. Electrochemical workstation was used to quickly characterize the corrosion resistance of graphene-modified anticorrosion coating, and the change of the electrochemical curve during soaking in $3.5 \% \mathrm{NaCl}$ was investigated every 5 hours. It was found that the application of rGO to modify the anticorrosion coating could improve the corrosion resistance of the anticorrosion coating, and as the soaking time increased, the corrosion resistance of graphene-modified anticorrosion coating changed regularly. The study results indicated that when the content of rGO was $0.4 \%$, the porosity of epoxy coating decreased from $1.54 \%$ to $0.33 \%$, the porosity dropped by an order of magnitude, and the self-corrosion voltage was relatively positive $(-0.72434 \mathrm{~V})$. The self-corrosion current density was the lowest $\left(1.948 \times 10^{-6} \mathrm{~A} / \mathrm{cm}^{2}\right)$, and at the low frequency, the impedance modulus was the highest $\left(10^{3}\right)$. After the equivalent circuit fitting, the dispersion index was relatively high, the dispersion effect was relatively weak, and the corrosion resistance of the coating was improved. For graphene-modified anticorrosion coating, in the early stage of corrosion protection, the existence of pores and other defects in the coating might increase the dispersion effect, resulting in greatly decreased corrosion resistance of the coating. In the middle stage of corrosion protection, the pores in the coating would be completely filled by corrosive ions, resulting in a weakened dispersion effect. Therefore, the decrease in the corrosion resistance of the coating was slowed down and became stable.
\end{abstract}

\section{Introduction}

Aluminum alloy has been widely used in aircraft frames, skins, fuel tanks, landing gear struts and other structures due to its low density, high specific strength, good ductility, good workability and other characteristics. The strong chemical activity of aluminum alloy makes it easy to form oxide film on the surface, but the film is thin (usually $10-15 \mathrm{~nm}$ ) and has uneven dispersion and poor compactness, leading to poor corrosion resistance of the film [1]. Therefore, it cannot play a good protective role. Especially in the marine atmospheric environment rich in $\mathrm{Cl}^{-}$, the higher concentration of corrosive ions can easily penetrate the oxide film, leading to corrosion of the aluminum alloy substrate [2]. A series of protective measures have been developed worldwide for serious metal corrosion. As the simplest, efficient, and economical anticorrosion measure, organic coating protection has attracted wide attention. Common organic coatings mainly include epoxy resin coating, polyurethane resin coating, and fluorocarbon resin coating. Epoxy resin is characterized by greater hardness and higher adhesion, but cured coating has large porosity and poor acid resistance and UV 
TABLE 1: 7075-T6 aluminum alloy composition.

\begin{tabular}{lcccccccccc}
\hline Component & $\mathrm{Zn}$ & $\mathrm{Mg}$ & $\mathrm{Cu}$ & $\mathrm{Si}$ & $\mathrm{Fe}$ & $\mathrm{Mn}$ & $\mathrm{Cr}$ & $\mathrm{Ti}$ & $\mathrm{Al}$ & Others \\
\hline Percentage & 5.6 & 2.5 & 1.4 & 0.35 & 0.42 & 0.29 & 0.22 & 0.1 & Allowance & 0.15 \\
\hline
\end{tabular}

resistance. Polyurethane resin has excellent flexibility, abrasion resistance, and mechanical properties, but its corrosion resistance will drop sharply after UV aging, which will seriously affect the protection of metallic substrate. Even though fluorocarbon resin has excellent UV resistance, its lower adhesion limits its future applications. Graphene is currently the lightest and thinnest material, and its microlamellar structure and small size effect have been found to be effective in improving the structure of the anticorrosion coating and increasing its corrosion resistance.

Graphene-modified anticorrosion coatings have the following characteristics:

(1) Light weight: the application of graphene to modify anticorrosion coatings can effectively reduce the thickness and improve the protection efficiency of coatings [3].

(2) Excellent barrier properties: graphene can be effectively filled in the micropores in coatings to improve the pores and other defects of anticorrosion coatings; moreover, the conjugated binary lamellar structure of graphene is superimposed in the coating to form a dense physical insulation layer, and the surface has excellent hydrophobic property after adsorbing alkanes and can greatly block the infiltration and penetration of water molecules and corrosive media into the coating [4].

(3) Excellent electrical conductivity: after the metallic substrate is corroded, it can quickly transfer electrons to the coating surface to prevent stacking of corrosion products on the substrate surface [5].

(4) Excellent mechanical properties: the C-C bond in the graphene structure makes graphene have good structural rigidity, which can effectively improve the flexibility and impact resistance of graphene-modified anticorrosion coatings [6].

To this end, researchers have conducted several studies [7-10]. Pourhashem et al. [11] used silane coupling agents APTES (KH550) and GPTMS (KH560) to prepare graphene oxide-modified epoxy resin coating and investigated its protective mechanism in stainless steel. Ye et al. [12] used covalent grafting to prepare functionalized graphene-modified epoxy resin coating and investigated its corrosion resistance in Q235 steel. Paul et al. [13] polymerized acrylic monomer solution to synthesize hydrophobic organosiloxane-acrylic resin and covalently bonded to fluorosilane-modified graphene nanosheets to prepare superhydrophobic $\left(\geq 152^{\circ}\right)$ anticorrosion coating, which improved the protection of LY12 aluminum alloy. In the above studies on graphene-modified anticorrosion coatings, graphene oxide (GO) was mostly used to mod- ify anticorrosion coatings, and the protection mechanism was investigated in stainless steel substrates. However, the effects of graphene-modified anticorrosion coatings on aviation aluminum alloy substrates were rarely investigated. Therefore, the following contents were investigated in this paper:

After using rGO to modify the epoxy primer, its porosity dropped by an order of magnitude. After cooperating with the graphene-modified polyurethane coating, its self-corrosion voltage is relatively positive, the selfcorrosion current density is low, the impedance modulus value is large at low frequency, and it has high corrosion resistance. With the extension of the immersion time in $3.5 \% \mathrm{NaCl}$ solution, the weakening of the dispersion effect decreases, and the corrosion resistance of the coating decreases slowly. The corrosion resistance mechanism of aviation aluminum alloy is explored.

\section{Experimental Section}

2.1. Materials. The metal substrate used in the experiment was 7075-T6 aluminum alloy, and its composition is shown in Table 1. The coatings were epoxy resin primer and polyurethane resin top coat provided by the Marine Chemical Research Institute in China. Reduced graphene oxide was 1133 anticorrosion graphene produced by The Sixth Element (Changzhou) Materials Technology Co., Ltd. in China. The graphene dispersant was F463 dispersant produced by Foshan Aona Polymer Co., Ltd. The other chemical reagents used in the experiment were purchased from Aladdin.

2.2. Preparation of $r G O$ Dispersion. Reduced graphene oxide, F463 dispersant, and xylene solution were mixed in a ratio of $1: 0.1: 10$ and then ultrasonically treated in a $600 \mathrm{~W}$ water bath sonicator for 2 hours to obtain the uniform graphene dispersion.

2.3. Preparation of Anticorrosion Coatings. Before coating, the surface of 7075-T6 aluminum alloy was sandblasted with 120-mesh white corundum, ultrasonically degreased with acetone, dehydrated with absolute ethyl alcohol, and finally dried and placed in a dry vessel for later use. Epoxy resin, curing agent, and diluent were mixed in a ratio of $8: 1: 3$, and then the prepared graphene dispersion was doped ( $\mathrm{rGO}$ addition ratio was $0.4 \% \mathrm{wt}$ ). The epoxy resin doped with reduced graphene oxide was magnetically stirred for 20 minutes. Atmospheric spray technology was used to spray on the sandblasted surface of aluminum alloy and cured at $70^{\circ} \mathrm{C}$ for 1 hour (air pressure was maintained at 6-7 MPa during spraying, and the spraying distance was maintained at 15$20 \mathrm{~cm}$ ). After the epoxy resin coating was completely dry, the above steps and addition ratio were repeated to prepare the polyurethane resin coating on the surface of epoxy resin primer. The total thickness of the anticorrosion coating was 


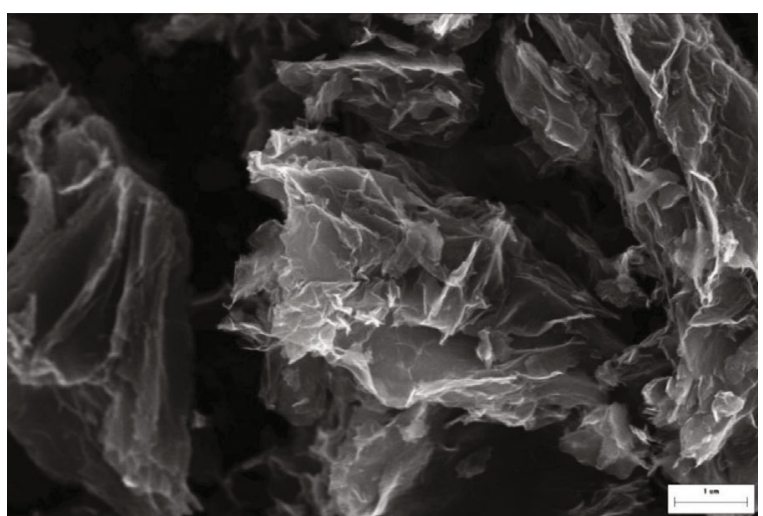

Figure 1: SEM image of rGO.

about $150 \mu \mathrm{m}$. The anticorrosion coating undoped with reduced graphene oxide was prepared in the same way as a blank control.

2.4. Characterization. A scanning electron microscope $(7900 \mathrm{~F})$ was used to observe the microscopic morphology of the cross-section of coating. Raman spectrometer (wavelength $532 \mathrm{~nm}$ ) was used to measure the Raman spectrum of the coating to investigate the microstructure of reduced graphene in the coating. Autolabe PGSTAT 302N electrochemical workstation (Metrohm, Switzerland) was used to determine the polarization curve and $\mathrm{AC}$ impedance of the anticorrosion coating and analyze the corrosion resistance of the anticorrosion coating and the change in the corrosion resistance of the graphene-modified anticorrosion coating after soaking in $3.5 \% \mathrm{NaCl}$. NOVA software was used to fit the electrochemical curve and draw the equivalent circuit. Before testing, it needed to be connected to a copper wire, and then, the sample was sealed with epoxy resin. The exposed coating area was fixed to $1 \mathrm{~cm}^{2}$. The electrochemical measurement was performed with a three-electrode system: a $10 \times 10 \mathrm{~mm}$ platinum sheet was used as the counter electrode, a saturated calomel electrode was used as the reference electrode, the test interval of the polarization curve was selfcorrosion potential $( \pm 150 \mathrm{mV})$, the scanning speed was $1 \mathrm{mV} / \mathrm{s}$, the frequency range of sine wave in the AC impedance test (EIS) was $0.01-100000 \mathrm{~Hz}$, and the amplitude of loaded AC disturbance voltage was $10 \mathrm{mV}$.

\section{Results and Discussion}

\subsection{Characterization of $r G O$}

3.1.1. SEM of $r G O$. At present, the main methods for preparation of graphene include mechanical exfoliation, chemical vapor deposition, $\mathrm{SiC}$ epitaxial growth, and redox process. The rGO used in this experiment was prepared by redox process, and its microscopic morphology is shown in Figure 1. It can be seen from Figure 1 that the rGO sheet had a large number of layers and the edges were wrinkled. The reason was that the hydroxyl groups (including the hydroxyl groups formed by the conversion of various oxygen-containing functional groups) on the benzene ring branches during the preparation of $\mathrm{rGO}$ by redox process, new $\mathrm{C}=\mathrm{C}$ double bonds were formed, and the electrostatic repulsion between graphene sheets was weakened, leading to intensified rGO agglomeration and increased edge defects [14, 15].

3.1.2. Dispersion of $r G O$. The comparison of rGO dispersion before and after standing for 20 days is shown in Figures 2(a) and 2(b) . It can be seen from Figures 2(a) and 2(b) that the color of rGO dispersion after standing for 20 days was not changed, and no rGO precipitation occurred, indicating that F463 dispersant can make rGO evenly dispersed in xylene. In the analysis of the dispersion mechanism, after ultrasound is used to predisperse rGO, the large van der Waals force of rGO makes its surface adsorb the chain-like polymer structure in the dispersant. And then relying on the physical barrier and electrostatic repulsion between polymers, the dispersion of rGO in the solution is achieved (as shown in Figure 2(c)).

The Raman spectrum of the prepared graphene-modified anticorrosion coating is shown in Figure 3. It could be seen from the figure that there were three characteristic peaks of graphene: D peak (near $1350 \mathrm{~cm}^{-1}$ ), G peak (near $1580 \mathrm{~cm}^{-1}$ ), and 2D peak (near $2690 \mathrm{~cm}^{-1}$ ). D peak was a graphene defect peak, which required at least one defect to activate. The higher intensity of $\mathrm{D}$ peak in Figure 3 indicated that there were more edges and defects in the graphene. The 2D peak of single-layer graphene was located near $2690 \mathrm{~cm}^{-1}$ and sharp, and its peak intensity was about 4 times that of $\mathrm{G}$ peak. As the number of graphene layers increased, its location shifted to the right, the peak intensity decreased, and the peak width increased $[16,17]$. When the graphene sheet reached ten layers or more, the shape of its $2 \mathrm{D}$ peak was basically the same as that of graphite. It was formed by the superposition of two peaks, and its intensity was about $1 / 4$ or $1 / 2$ of the $G$ peak intensity, respectively. As shown in Figure 2, $I_{2 \mathrm{D}}: I_{\mathrm{G}}$ was about $1: 1$, indicating that the dopant was graphene with more layers rather than graphite [18]. The grain size of rGO in the graphene-modified anticorrosion coating was about $12 \mathrm{~nm}$, calculated by formula (1) [19] for graphene grain size.

$$
\mathrm{La}(\mathrm{nm})=\left(2.4 \times 10^{-10}\right) \lambda^{4}\left(\frac{I_{D}}{I_{G}}\right)^{-1},
$$

where $\lambda$ is the wavelength of the excitation light source $(532 \mathrm{~nm})$.

3.2. Morphology of Anticorrosion Coatings. The crosssectional micromorphology of original and graphenemodified anticorrosion coatings is shown in Figure 4. As can be seen from Figure 4, epoxy resin has excellent chemical stability and adhesion; it not only has anticorrosion effect on the aluminum alloy substrate but also strengthens the bonding of anticorrosion coating to aluminum alloy substrate. However, with the volatilization of diluent during hightemperature curing, more pores may occur. Pores are typical defects in anticorrosion coatings. As the corrosion progresses, corrosive ions enter the pores and defects of coatings 


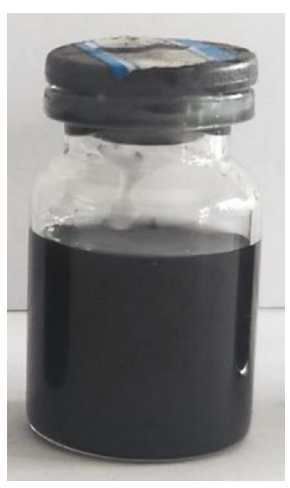

(a)

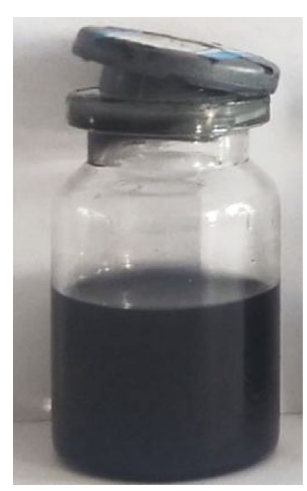

(b)
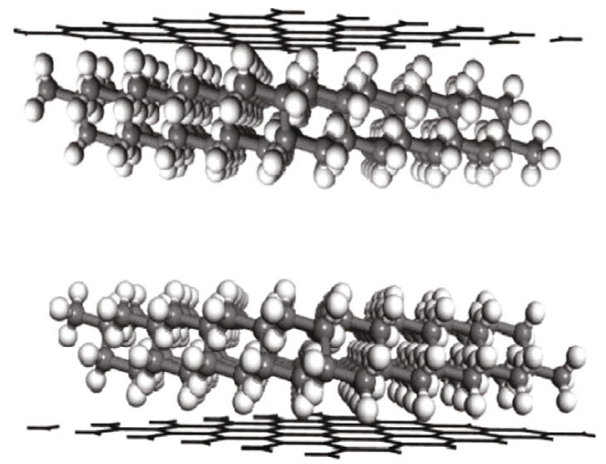

(c)

Figure 2: Dispersion of rGO ((a) 0d; (b) 20d; (c) dispersion mechanism).

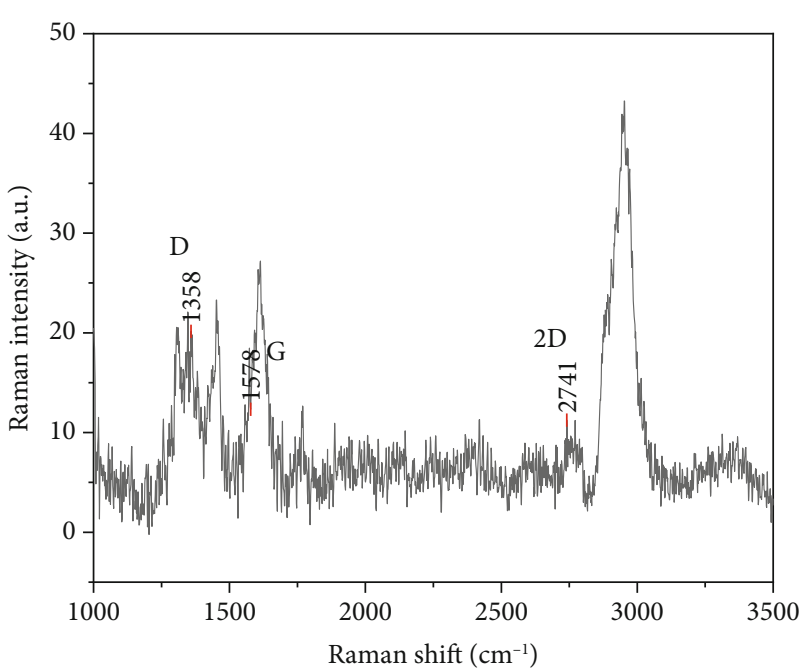

FIGURE 3: Raman of graphene-modified coating.

through diffusion, forming an ion pathway connecting the metal substrate with the outside. This not only destroys organic coatings but also accelerates the corrosion of the metal substrate $[20,21]$. Polyurethane resin has low viscosity and good fluidity. The top coat formed after hightemperature curing has no large porosity, and corrosive media such as $\mathrm{Cl}^{-}$and water cannot pass through easily. Therefore, Image-Pro software was used to calculate the porosity in epoxy resin primer (as shown in Table 2). From the data in Table 2, it could be seen that the porosity in the epoxy resin primer in graphene-modified anticorrosion coating was one order of magnitude lower than that of the original coating. And the anticorrosion coating defects were significantly improved. This is because the predispersed rGO surface adsorbs more organic polymers and the rGO is effectively dispersed after it is fully stirred with the polymer diluent. In the subsequent curing process, as the diluent volatilizes, rGO occupies the volatilized dilution. The location where the agent is located and relying on its own excellent microlamella structure and small size effect effectively prevent the penetration of corrosion ions $\left(\mathrm{Cl}^{-}\right)$into the anticorrosion coating and reduce the formation of "water vapor pathway," thereby enhancing the corrosion resistance of coatings.

3.3. Electroactivity of Anticorrosion Coatings. The polarization curve of original and graphene-modified anticorrosion coatings is shown in Figure 5. Self-corrosion potential $\left(E_{\text {corr }}, \mathrm{SCE}\right)$ indicates the degree of difficulty of corrosion of the test sample, while self-corrosion current density $\left(J_{\text {corr }}\right)$ is a favorable basis for quickly evaluating the corrosion resistance of coating. It could be seen from Figure 5 that when graphene-modified anticorrosion coating was compared with original anticorrosion coating, the selfcorrosion potential was relatively positive. This indicates that graphene-modified anticorrosion coating is not prone to corrosion, and the addition of rGO hinders the anodic dissolution and cathodic formation occurring in oxide film on the aluminum alloy surface during polarization. 


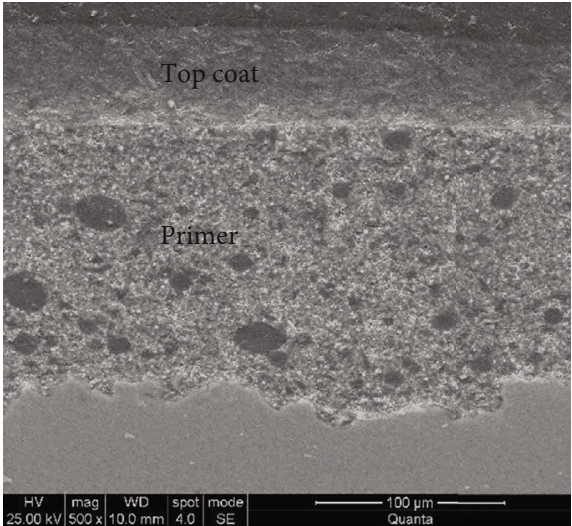

(a)

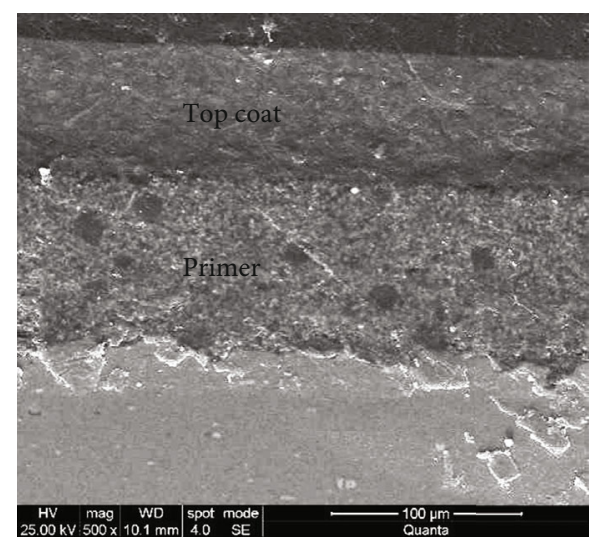

(b)

FIGURE 4: SEM images of original and graphene-modified coatings ((a) original; (b) graphene-modified coating).

TABLE 2: Porosity of original and graphene-modified coatings.

\begin{tabular}{lcc}
\hline Coatings & Original & Graphene-modified coating \\
\hline Porosity (\%) & 1.54 & 0.33 \\
\hline
\end{tabular}

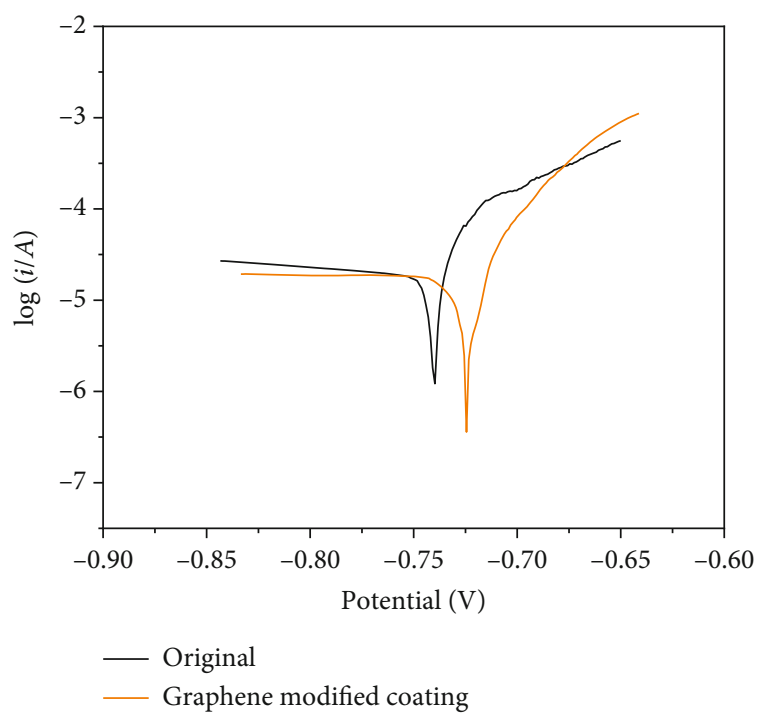

FIGURE 5: Tafel of original and graphene-modified coatings.

The results of the Tafel curve fitting using Nova software are shown in Table 3 , where $b_{a}$ is the Tafel slope of the anode, $b_{c}$ is the Tafel slope of the cathode, and $R_{p}$ is the polarization resistance calculated according to formula (2). It could be seen from the fitting data in Table 3 that the self-corrosion current density on graphene-modified anticorrosion coating decreased and the polarization resistance increased. The addition of rGO enlarged the transmission route of electrons in the anticorrosion coating, and the electrical resistance of the coating was increased, thereby improving the corrosion resistance of the coating.

$$
R_{p}=\frac{b_{a} / b_{c}}{2.303\left(b_{a}+b_{c}\right) J_{\text {coor }}}
$$

The Bode curve of original and graphene-modified anticorrosion coatings is shown in Figure 6 ((a) impedance modulus diagram and (b) phase angle diagram). In the electrochemical corrosion test, the impedance modulus $(|Z|)$ in the low-frequency region was used to compare the corrosion resistance of coatings. In Figure 6(a), $|Z|$ of graphene-modified anticorrosion coating at low frequency $>103>|Z|$ of original anticorrosion coating at low frequency indicates that graphene-modified anticorrosion coating has better corrosion resistance. This was consistent with the result shown in the polarization curve.

The Nyquist curve of the anticorrosion coating obtained by the electrochemical workstation is shown in Figure 7 . The size of capacity reactance arc radius in the Nyquist curve indicates the diffusion speed of corrosion products and the easiness of charge transfer. The comparison of the capacity reactance arc radius in Figure 7 showed that corrosion products produced on the surface of aluminum alloy protected by graphene-modified anticorrosion coating were not easy to stack, the charge was easily transferred, and the corrosion of the aluminum alloy substrate was inhibited. In the original and graphene-modified anticorrosion coatings in Figure 7, there was inductive reactance arc in addition to capacity reactance arc. The appearance of inductive reactance arc was caused by the destruction of oxide film on the aluminum alloy surface, thereby activating the pitting corrosion [22]. In the phase angle curve in Figure 6(b), both the anticorrosion coatings had a peak and a trough. The equivalent circuit diagram was drawn according to the Nyquist curve, as shown in Figure 8 (where $R_{s}$ is the solution resistance, $Q_{\mathrm{dl}}$ is the constant phase angle element, $R_{\mathrm{ct}}$ is the coating resistance, $L$ is the inductance caused by pitting corrosion, and $R_{m}$ is the oxide film resistance), and the data obtained by fitting the elements in the equivalent circuit with software are shown in Table 4 [23]. In Table $4, R_{c t}$ of graphene-modified anticorrosion coating was doubled compared with that of original anticorrosion coating. Graphene-modified anticorrosion coating had higher dispersion index and larger dispersion 
TABLE 3: Results of Tafel curve fitting.

\begin{tabular}{lccccr}
\hline & $E_{\text {corr }}(\mathrm{V})$ & $J_{\text {corr }}\left(\mathrm{A} / \mathrm{cm}^{2}\right)$ & $b_{a}$ & $b_{c}$ & $R_{p}(\Omega)$ \\
\hline Original & -0.74045 & $3.801 \times 10^{-6}$ & 0.0081057 & 0.010076 & 510.68 \\
Graphene-modified coatings & -0.72434 & $1.948 \times 10^{-6}$ & 0.0073826 & 0.010325 & 1014.5 \\
\hline
\end{tabular}

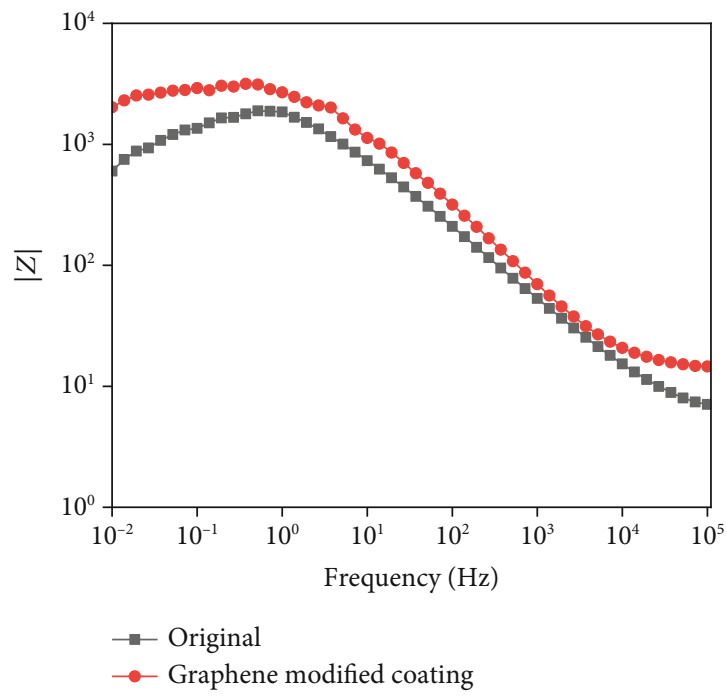

(a)

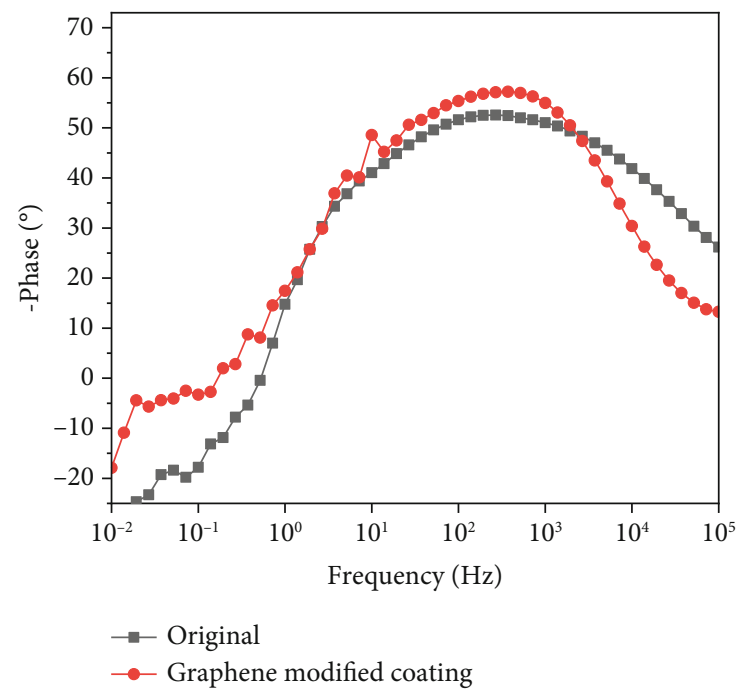

(b)

Figure 6: Bode of the original and graphene-modified coatings ((a) impedance modulus curve; (b) phase angle curve).

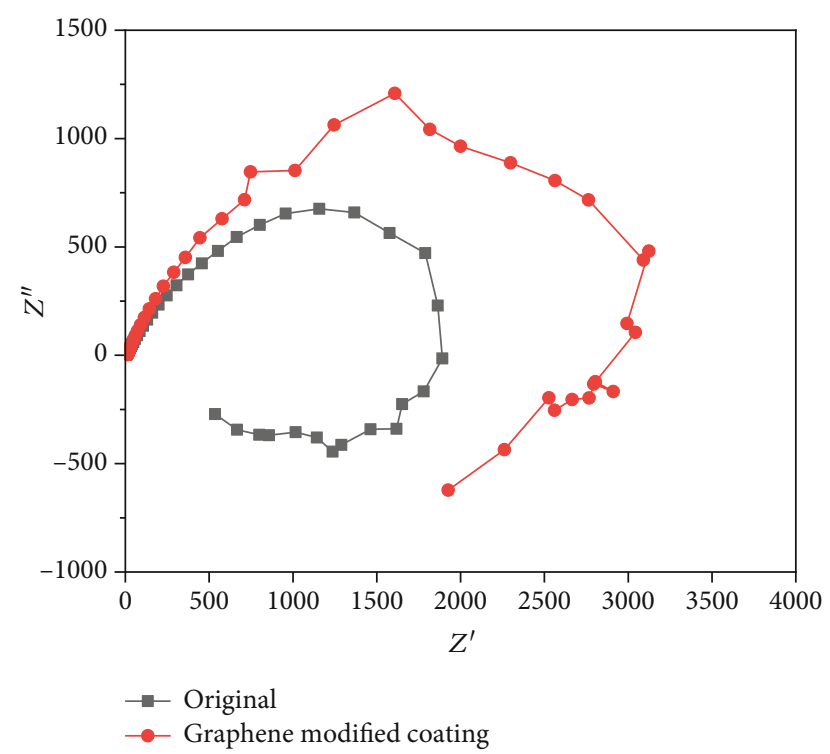

FIGURE 7: Nyquist of original and graphene-modified coatings.

effect. The dispersion index tended to be 1 . The surface of graphene-modified anticorrosion coating tended to be an electrical double-layer capacitor and had strong corrosion resistance [24].

The Bode curve of graphene-modified anticorrosion coating soaked in $3.5 \% \mathrm{NaCl}$ for different times is shown in Figure 9. The equivalent circuit drawn according to

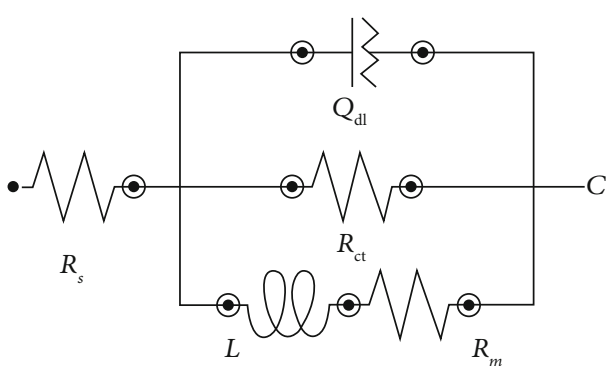

Figure 8: Equivalent circuit diagram.

TABLe 4: Fitting data of equivalent circuit components.

\begin{tabular}{lcccc}
\hline Coatings & $R_{s}(\Omega)$ & $R_{c t}(\Omega)$ & $Y_{0}$ & $N$ \\
\hline Original & 5.467 & 1067.2 & $5.071 \times 10^{-5}$ & 0.679 \\
Graphene-modified coating & 12.922 & 2768 & $3.076 \times 10^{-5}$ & 0.716 \\
\hline
\end{tabular}

Figure 8(b) (phase angle curve) and Figure 10 (Nyquist curve) was consistent with that Figure 8, and the fitting data of equivalent circuit components are shown in Table 5. In the impedance modulus curve shown in Figure 9(a), it could be seen that the $|Z|$ value did not change at low frequency in the early stage of soaking, and the corrosion resistance of graphene-modified anticorrosion coating did not decrease. After soaking for 10 hours, at low frequency, the $|Z|$ value 


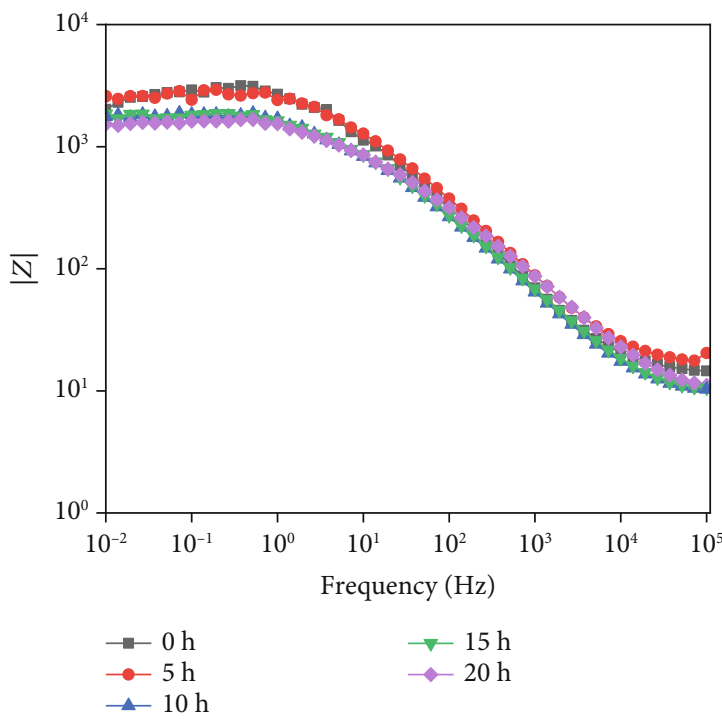

(a)

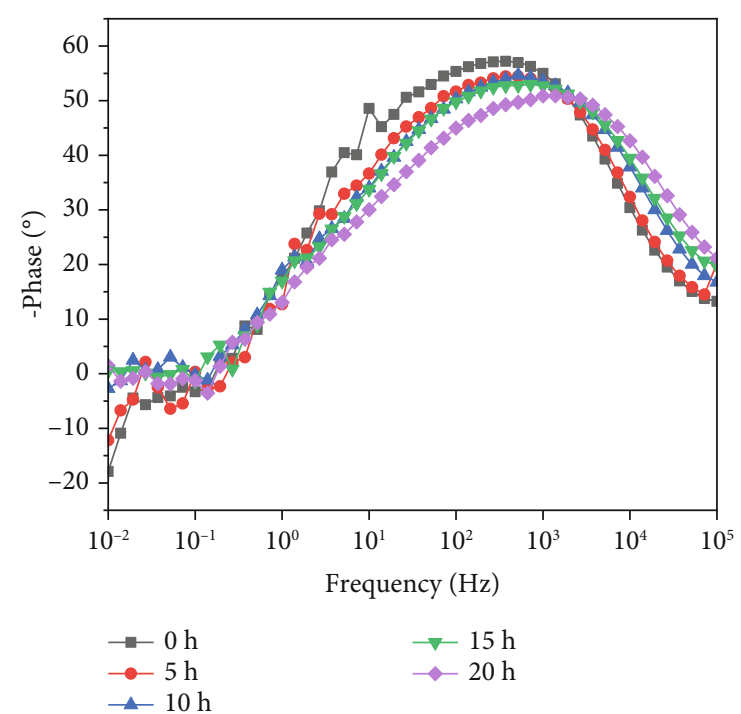

(b)

FIGURE 9: Bode of graphene-modified anticorrosion coating soaked in $3.5 \% \mathrm{NaCl}$ for different times ((a) impedance modulus curve; (b) phase angle curve).

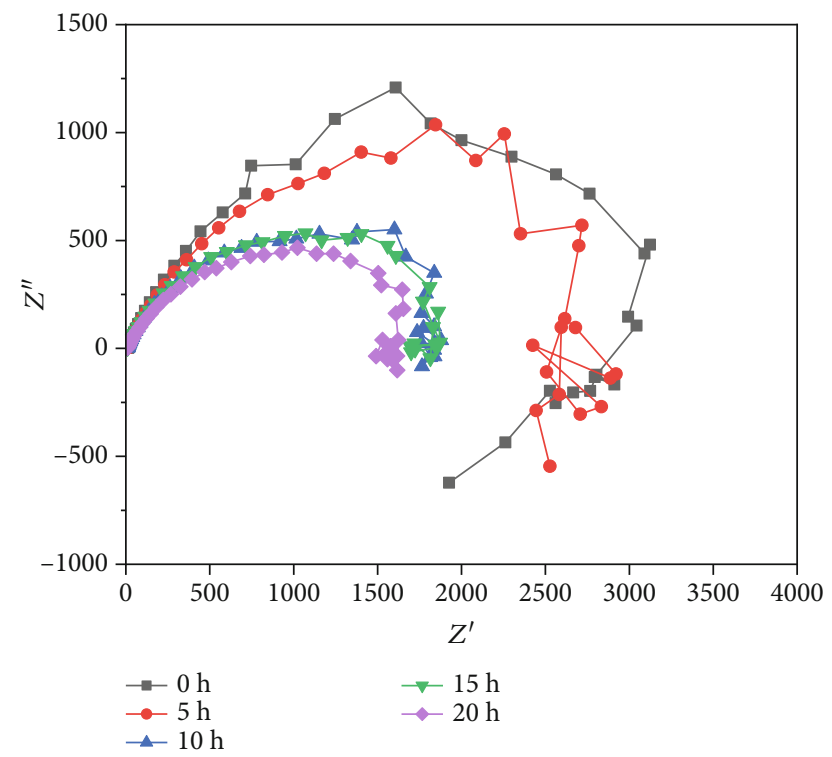

FIgURE 10: Nyquist of graphene-modified anticorrosion coating soaked in $3.5 \% \mathrm{NaCl}$ for different times.

TABLE 5: Fitting data of equivalent circuit components.

\begin{tabular}{lcccc}
\hline Time & $R_{s}(\Omega)$ & $R_{c t}(\Omega)$ & $Y_{0}$ & $N$ \\
\hline $0 \mathrm{~h}$ & 12.922 & 3768 & $3.076 \times 10^{-5}$ & 0.716 \\
$5 \mathrm{~h}$ & 14.82 & 2734.9 & $3.025 \times 10^{-5}$ & 0.690 \\
$10 \mathrm{~h}$ & 7.786 & 1861.9 & $5.128 \times 10^{-5}$ & 0.661 \\
$15 \mathrm{~h}$ & 7.574 & 1851.4 & $5.0753 \times 10^{-5}$ & 0.6548 \\
$20 \mathrm{~h}$ & 7.105 & 1638.2 & $5.152 \times 10^{-5}$ & 0.6261 \\
\hline
\end{tabular}

decreased to a large extent, and the corrosion resistance of graphene-modified anticorrosion coating decreased greatly. Then, as the soaking time increased, the decrease of the $|Z|$ value at low frequency slowed down. The capacity reactance arc radius in the Nyquist curve and the dispersion index in the fitted data showed the same change. In the analysis of reason, corrosive ions were gradually filled into the pores and other defects of graphene-modified anticorrosion coating within 5 hours of soaking, thus realizing the invasion of corrosive ions to the surface of aluminum alloy. And then, as the pores and other defects were completely filled by corrosive ions, the dispersion effect of corrosive ions in graphene-modified anticorrosion coating decreased and became stable. The corrosive ions gradually destroyed the graphene-modified anticorrosion coating, and the area of corrosive ions in the graphenemodified anticorrosion coating was expanded. This reduces the corrosion resistance of the graphene-modified anticorrosion coating [25].

\section{Conclusions}

(1) rGO was used to prepare graphene-modified anticorrosion coating, and the cross-sectional micromorphology of graphene-modified anticorrosion coating and the microstructure of rGO in the coating were tested and analyzed. It is found that rGO could effectively improve the porosity of graphene-modified anticorrosion coating by relying on its microlamellar structure and small size effect and reduce it by an order of magnitude. rGO was prone to reagglomeration in the anticorrosion coating, and the rGO sheet in the graphene-modified anticorrosion coating reached more than 10 layers 
(2) The electrochemical performance of anticorrosion coating was tested and analyzed, and it was found that compared with the original anticorrosion coating, the self-corrosion voltage of the graphenemodified anticorrosion coating was relatively positive, the self-corrosion current density was relatively low, the polarization resistance was relatively large, the impedance modulus at low frequency was relatively large, the dispersion effect in the equivalent circuit components was relatively weak, and the coating had better corrosion resistance

(3) The electrochemical curve of the graphene-modified anticorrosion coating soaked in $3.5 \% \mathrm{NaCl}$ for different times was tested and analyzed, and it was found that corrosive ions showed a strong dispersion effect in a period of time due to the existence of pores and other defects in the graphene-modified anticorrosion coating in the early stage of soaking. As the pores and other defects were completely filled, the dispersion effect slowed down and became stable, thereby reducing the corrosion resistance of the graphene-modified anticorrosion coating

\section{Data Availability}

The data used to support the findings of this study are available from the corresponding author upon request.

\section{Conflicts of Interest}

The authors declare that they have no conflicts of interest.

\section{References}

[1] V. K. Beura, C. Kale, S. Srinivasan, C. L. Williams, and K. N. Solanki, "Corrosion behavior of a dynamically deformed Al-Mg alloy," Electrochimica Acta, vol. 354, p. 136695, 2020.

[2] M. A. Wahid, A. N. Siddiquee, and Z. A. Khan, "Aluminum alloys in marine construction: characteristics, application, and problems from a fabrication viewpoint," Marine Systems \& Ocean Technology, vol. 15, no. 1, pp. 70-80, 2020.

[3] A. K. Geim and K. S. Novoselov, "The rise of graphene," Nature Materials, vol. 6, no. 3, pp. 183-191, 2007.

[4] H. Wu and L. T. Drzal, "Graphene nanoplatelet paper as a light-weight composite with excellent electrical and thermal conductivity and good gas barrier properties," Carbon, vol. 50, no. 3, pp. 1135-1145, 2011.

[5] P. Dhiraj, T. J. Carlos, R. Harl Robert, J. G. Kane, R. Rogers Bridget, and I. Bolotin Kirill, "Graphene: corrosion-inhibiting coating," ACS Nano, vol. 6, no. 2, pp. 1102-1108, 2012.

[6] M. A. Krishnan, K. S. Aneja, A. Shaikh et al., "Graphene-based anticorrosive coatings for copper," RSC Advances, vol. 8, no. 1, pp. 499-507, 2018.

[7] C. Cheng, S. Qiu, M. Cui et al., "Achieving high performance corrosion and wear resistant epoxy coatings via incorporation of noncovalent functionalized graphene," Carbon, vol. 114, pp. 7858-7862, 2017.

[8] Y. T. Hui, Z. Z. Hua, L. Jin et al., "Corrosion protection of aluminum alloy by epoxy coatings containing polyaniline modi- fied graphene additives," Materials and Corrosion, vol. 70, no. 7, pp. 1298-1305, 2019.

[9] S. Lu, Y. Li, W. Zhao et al., "Tuning F-doped degree of rGO: restraining corrosion-promotion activity of $\mathrm{EP} / \mathrm{rGO}$ nanocomposite coating," Journal of Materials Science \& Technology, vol. 44, no. 9, pp. 121-132, 2020.

[10] H. Wang, L. Grabstanowicz, H. Barkholtz, D. Rebollar, and D.-J. Liu, "Impacts of imidazolate ligand on performance of zeolitic-imidazolate framework-derived oxygen reduction catalysts," ACS Energy Letters, vol. 4, no. 10, pp. 2500 2507, 2019.

[11] S. Pourhashem, A. Rashidi, M. R. Vaezi, and M. R. Bagherzadeh, "Excellent corrosion protection performance of epoxy composite coatings filled with amino-silane functionalized graphene oxide," Surface \& Coatings Technology, vol. 317, pp. 1-9, 2017.

[12] Y. Ye, D. Zhang, T. Liu et al., "Superior corrosion resistance and self-healable epoxy coating pigmented with silanzied trianiline-intercalated graphene," Carbon, vol. 142, pp. 164176, 2018.

[13] P. C. Uzoma, F. Liu, L. Xu et al., "Superhydrophobicity, conductivity and anticorrosion of robust siloxane-acrylic coatings modified with graphene nanosheets," Progress in Organic Coatings, vol. 127, pp. 239-251, 2019.

[14] C. C. Kiang and P. Martin, "Chemical reduction of graphene oxide: a synthetic chemistry viewpoint," Chemical Society reviews, vol. 43, no. 1, pp. 291-312, 2014.

[15] S. Stankovich, D. A. Dikin, R. D. Piner et al., "Synthesis of graphene-based nanosheets via chemical reduction of exfoliated graphite oxide," Carbon, vol. 45, no. 7, pp. 1558-1565, 2007.

[16] R. Thomsen, "Double resonant Raman scattering in graphite," Physical Review Letters, vol. 85, no. 24, pp. 52145217, 2000.

[17] N. T. An, L. Jae-Ung, Y. Duhee, and C. Hyeonsik, "Excitation energy dependent Raman signatures of ABA- and ABCstacked few-layer graphene," Scientific reports, vol. 4, no. 7, article 4630, 2014.

[18] R. J. Nemanich and S. A. Solin, "First- and second-order Raman scattering from finite-size crystals of graphite," Physical Review B, vol. 20, no. 2, pp. 392-401, 1979.

[19] L. G. Cancado, K. Takai, T. Enoki et al., "General equation for the determination of the crystallite size La of nanographite by Raman spectroscopy," Applied Physics Letters, vol. 88, no. 16, 2006.

[20] A. Meroufel, C. Deslouis, and S. Touzain, "Electrochemical and anticorrosion performances of zinc-rich and polyaniline powder coatings," Electrochimica Acta, vol. 53, no. 5, pp. 2331-2338, 2007.

[21] E. Rocca, C. Juers, and J. Steinmetz, "Corrosion behaviour of chemical conversion treatments on as-cast $\mathrm{Mg}-\mathrm{Al}$ alloys: electrochemical and non-electrochemical methods," Corrosion Science, vol. 52, no. 6, pp. 2172-2178, 2010.

[22] A. Venugopal and V. S. Raja, "AC impedance study on the activation mechanism of aluminium by indium and zinc in $3.5 \% \mathrm{NaCl}$ medium," Corrosion Science, vol. 39, no. 12, pp. 2053-2065, 1997.

[23] G. Bierwagen, D. Tallman, J. Li, L. He, and C. Jeffcoate, "EIS studies of coated metals in accelerated exposure," Progress in Organic Coatings, vol. 46, no. 2, pp. 149-158, 2003. 
[24] J. D. Scantlebury and K. Galić, "The application of AC impedance to study the performance of lacquered aluminium specimens in acetic acid solution," Progress in Organic Coatings, vol. 31, no. 3, pp. 201-207, 1997.

[25] S. Syed, "Influence of the environment on atmospheric corrosion of aluminiumCorrosion engineering," Science and Technology, vol. 45, no. 4, pp. 282-287, 2010. 